\title{
1 Bootstrat: Population Informed Bootstrapping for Rare Variant
}

\section{Tests}

4 Hailiang Huang ${ }^{1,2^{*}}$, Gina M. Peloso ${ }^{3}$, Daniel Howrigan ${ }^{1,2}$, Barbara Rakitsch ${ }^{4,5}$, Carl Johann

5 Simon-Gabriel ${ }^{5}$, Jacqueline I. Goldstein ${ }^{1,2}$, Mark J. Daly ${ }^{1,2}$, Karsten Borgwardt ${ }^{4,5,6,7}$,

6 Benjamin M. Neale ${ }^{1,2^{*}}$

8 1. Analytical and Translational Genetics Unit, Department of Medicine, Massachusetts

9 General Hospital and Harvard Medical School, Boston, MA 02114, USA

10 2. Stanley Center for Psychiatric Research, Broad Institute of MIT and Harvard,

11 Cambridge, MA 02142, USA

12 3. Center for Human Genetic Research, Massachusetts General Hospital, Boston MA

13 02114, USA

14 4. Machine Learning and Computational Biology Research Group, Max Planck Institute

15 for Developmental Biology and Max Planck Institute for Intelligent Systems, 72076

16 Tübingen, Germany

17 5. Center for Bioinformatics, Eberhard Karls Universität Tübingen, 72076 Tübingen,

18 Germany

19 6. Machine Learning and Computational Biology Lab, Department Biosystems Science

20 and Engineering, ETH Zürich, 4058 Basel, Switzerland

21 7. SIB Swiss Institute of Bioinformatics, 4058 Basel, Switzerland

22

23

24 Emails: bneale@broadinstitute.org (BMN) and hhuang@broadinstitute.org (HH) 


\section{Abstract}

26 Recent advances in genotyping and sequencing technologies have made detecting rare

27 variants in large cohorts possible. Various analytic methods for associating disease to

28 rare variants have been proposed, including burden tests, C-alpha and SKAT. Most of

29 these methods, however, assume that samples come from a homogeneous population,

30 which is not realistic for analyses of large samples. Not correcting for population

31 stratification causes inflated $p$-values and false-positive associations. Here we propose a

32 population-informed bootstrap resampling method that controls for population

33 stratification (Bootstrat) in rare variant tests. In essence, the Bootstrat procedure uses

34 genetic distance to create a phenotype probability for each sample. We show that this

35 empirical approach can effectively correct for population stratification while maintaining

36 statistical power comparable to established methods of controlling for population

37 stratification. The Bootstrat scheme can be easily applied to existing rare variant testing

38 methods with reasonable computational complexity. 


\section{Author Summary}

40 Recent technology advances have enabled large-scale analysis of rare variants, but

41 properly testing rare variants remains a significant challenge as most rare variant testing

42 methods assume a sample of homogenous ethnicity, an assumption often not true for

43 large cohorts. Failure to account for this heterogeneity increases the type I error rate.

44 Here we propose a bootstrap scheme applicable to most existing rare variant testing

45 methods to control for population heterogeneity. This scheme uses a randomization

46 layer to establish a null distribution of the test statistics while preserving the sample

47 genetic relationships. The null distribution is then used to calculate an empirical $p$-value

48 that accounts for population heterogeneity. We demonstrate how this scheme

49 successfully controls the type I error rate without loss of statistical power. 


\section{Introduction}

52 Case-control association studies have successfully identified significant genetic

53 associations across a wide range of phenotypes including inflammatory bowel diseases

54 [1-3], cardiovascular diseases [4-7], and psychiatric diseases [8-11]. For complex traits,

55 the vast majority of significant associations have been largely restricted to common

56 variants (minor allele frequency $>1 \%$ ). In most instances, identification of causal

57 variants is challenging because of pervasive linkage disequilibrium among common

58 variants in a region. Analysis of rare variants has the potential to nominate additional

59 regions of interest; in particular coding variation holds promise for identifying specific

60 genes of interest. The advent of exome chip and advances in whole exome and genome

61 sequencing allow for high throughput detection and genotyping of rare variants.

62 Finding rare variants underlying human disorders, however, presents a significant

63 challenge due to the limited statistical power when only a small number of individuals

64 carry a given variant.

65 Several approaches have been designed to improve statistical power through

66 variant aggregation, patterns of observed variation, and mixture models [12-15];

67 however no approach is inherently designed to control for the confounding of

68 population stratification. Stratification is not an issue when datasets come solely from a

69 single homogenous population, but can become severe as dataset sizes increase and are

70 combined across multiple cohorts. For example, a recent study collected roughly 35,000

71 European samples across multiple cohorts and had to use 15 principal components (PCs)

72 to correct for population stratification $[2,16]$. Furthermore, stratification of rare 
73 variants may operate on a finer population scale than is easily correctable using current

74 methods [17]. Not appropriately adjusting for stratification can lead to inflated test

75 statistics and false-positive associations, limiting the usage of these methods.

76 Population stratification in common variant association tests can be controlled

77 by using genetic principal component analysis (PCA) [8] or linear mixed models (LMM)

$78[18,19]$. PCA models ancestry differences among individuals using principal components

79 of thousands of genetic markers across the genome, which are then used as covariates

80 in association tests. LMM uses the random effect model to estimate the contribution

81 from the genetic relationship matrix (GRM) to phenotypic variance, and adjusts the

82 association test statistics accordingly. While both methods work well for common

83 variant association tests, extending them to rare variants, if possible, is not obvious

84 because of their complexity.

85 Several existing methods have the potential to be adapted to control the

86 population stratification in rare variant association tests but they each have their

87 pitfalls. The genomic control method [20] adjusts the association test statistics using

88 the inflation factor estimated from unlinked variants. Despite its simplicity, the

89 assumption that the inflation factor is constant across the genome is unlikely to be true

90 especially for rare variants, therefore limiting the use of this method. Pritchard et al.

91 proposed a model-based clustering approach to infer the population structure [21] and

92 took advantage of this structure to perform randomizations for calculating the

93 significance of associations [22,23]. This method can be retrofit to existing rare variant

94 tests by clustering individuals of close genetic distance into a subgroup and shuffling 
95 phenotypes uniformly with in each group (discussed in Results and Methods). However,

96 this approach is not optimal because genetic relatedness falls along a broad continuum

97 and can be hard to categorize into a finite number of subgroups. In addition, some

98 clusters may only have cases or controls and thus have to be dropped, resulting in a

99 smaller effective sample size. A more sophisticated approach [24] used the Fisher's

100 non-central hypergeometric distribution [25] to resample disease status such that the

101 odds of a subject being selected as a case are equal to his or her odds of disease

102 conditional on confounder variables. Because disease status was used to train the

103 model, a potential problem is that the empirical distribution of test statistics can be

104 biased, leading to a biased empirical $p$-value (discussed in Results).

105 We propose an alternative approach, Bootstrat, that addresses the limitations of

106 the aforementioned methods using a bootstrap based randomization scheme informed

107 by the magnitude of genetic distance learned from the dataset. This scheme assigns

108 higher probabilities of sampling phenotypes from genetically related individuals. Unlike

109 permutation within discrete clusters, we are able to include all samples in the

110 randomization procedure. The randomization process is driven only by the genetic

111 similarity and has the potential to deal with non-monotonic effects across ancestry

112 space. When applied to a simulated dataset, Bootstrat successfully controls the

113 genomic inflation due to population stratification in both single variant and gene-based

114 tests, while keeping similar computation efficiency. In gene-based tests, Bootstrat

115 shows improved type I error control over other methods while maintaining the same

116 statistical power. 


\section{Results}

\section{Dataset}

119 All analyses in this study were performed on a dataset of 2,000 samples. Half of the

120 samples $(1,000)$ were randomly selected from an American control cohort and the other

121 half were randomly selected from a Swedish control cohort (Methods::Dataset). To

122 simulate the effect of population structure, we randomly selected $70 \%$ of American

123 samples and $30 \%$ of Swedish samples as cases. The remaining samples were labeled as

124 controls. For gene-based analyses, we selected variants with a minor allele frequency <

$1255 \%$ and labeled as non-synonymous, splice-site, or nonsense. Genes with at least 40

126 minor alleles were used for assessment. This threshold was chosen to allow good

127 asymptotic behavior and enough statistical power [26]. There were 5,725 genes and

12844,392 variants available for analysis. The number of minor alleles per gene ranges from

12940 to 3,506 with mean of 146 and median of 105.

\section{Bootstrat}

131 Typically, to empirically evaluate the significance of a hypothesis, a permutation test can

132 be used to construct a null distribution of the test statistics. The permutation test is

133 non-parametric (i.e. distribution free) and can provide an exact significance level.

134 However, it has an important assumption that all samples are exchangeable under the

135 null hypothesis [27], i.e., the joint probability distribution of the permuted sequence is

136 the same as that of the original sequence. This assumption holds in a homogenous

137 population where samples have equal probabilities to permute with each other. In a

138 stratified dataset, this assumption no longer holds because permutations between 
139 samples of the same ethnicity have higher exchange probabilities than those between

140 samples of different ethnicities. For example, in a dataset with mostly European

141 samples, if A has a nontrivial amount of Asian ancestry composition and B is almost

$142100 \%$ European, the probability for A to exchange with B would be higher than that for B

143 to exchange with $A$. This is because sample $B$ is more likely to exchange with a sample

144 of his/her own ancestry but sample A can only exchange with samples in other

145 ancestries. Therefore, the probability for sample A to shuffle with sample B can be

146 different from the probability for sample B to shuffle with sample A.

147 When this happens, bootstrap can be used in place of the permutation test to

148 evaluate the significance of a hypothesis. Weighted bootstrap $[1-3,28]$ relaxes the

149 exchangeability condition [1,3-7] by weighting each sample in the original dataset and

150 resampling them randomly with replacement. Therefore, we use weighted bootstrap,

151 instead of the permutation with uniform probabilities, to resample phenotypes and

152 create a null distribution of the association test statistics with awareness to population

153 stratification. The weighting reflects the genetic distances between samples, such that a

154 sample is more likely to draw a phenotype from samples sharing the similar ethnicity.

155 This is intuitively illustrated in Fig 1, which shows two samples shuffle only with other

156 samples that are genetically close. Once the null distribution corrected for population

157 stratifications is established, it can be used to calculate the empirical significance of the

158 observed test statistic.

159 Measure of population stratification 
160 In a GWAS with no confounding effects (e.g. no population stratification), most variants

161 are not associated with the trait and their test statistics follow the null distribution.

162 Presence of confounding effects inflates the test statistics in the study and causes them

163 to deviate from the null distribution. The Quantile-Quantile (QQ) plot compares the

164 observed quantiles versus the expected quantiles under the null hypothesis and is

165 frequently used to check that the distribution of statistics follows expectation. For

166 common variants, a well-behaved QQ plot only departs from the diagonal line at the tail,

167 representing the small proportion of significant associations. In addition to the QQ plot,

168 the genomic inflation factor, usually denoted as $\lambda$, quantitatively measures the deviation

169 of the observed distribution from the null distribution. $\lambda$ is defined as the ratio of the

170 median test statistic from the study over the median test statistic from the null

171 distribution and is expected to be 1 for common variants when there is no population

172 stratification. Both QQ plot and the genomic control factor work well for common

173 variants in measuring population stratification, but are not optimal for rare variants,

174 which usually have different asymptotic behaviors and will be discussed in later

175 sections. Unless otherwise noted, the test statistic used in this study is from the single

176 degree of freedom chi-square test using single variant logistic regression.

177 Principal components

178 The first principal component (PC) was able to identify the two population cohorts in our

179 dataset (Fig 1), and controls the population stratification in the QQ plot (S1 Fig) with a

180 reduced genomic inflation factor $(\lambda)$ from 1.330 to 1.000 . Increasing the number of PCs

181 to 2 and 3 has $\lambda=1.010$ and 0.992 , respectively. Thus, using more than the first PC does 
182 not improve our control over population stratification effects, and hurts statistical

183 power [4,8-11]. We therefore use only the first PC throughout this study.

\section{Estimate the Bootstrat parameter}

185 Bootstrat has a parameter, $\gamma$, that controls the magnitude of the correction. It

186 resembles the grid size or the number of clusters in the "permutation within cluster"

187 approach (See Methods). A larger $y$ results in a stronger control of stratification and

188 should be used in a severely stratified dataset. We estimate the optimal $\gamma$ by doing a

189 grid search to find the smallest $\gamma$ that sufficiently controls the population stratification

190 (S2 Fig). We note that further increasing $v$ beyond the optimal value resembles further

191 dividing population homogenous groups into smaller subgroups, which does not achieve

192 better population control and results in lower power. The desired level of genomic

193 control can be variable depending on many factors such as sample size and genotyping

194 chip. We took the genomic control factor from common variant association tests using

195 PC as covariates as the desired level. In the grid search, we allowed $\lambda$ to fall within the

$1961 \%$ range of this desired level. In this study, the desired level of $\lambda$ is 1.000 (Table 1) and

197 we allow $\lambda$ to range between 1.010 and 0.990 . We calculated $\lambda$ for $\gamma$ between -3 and 8

198 using common variants (Table 1). We found Bootstrat using $\gamma=4$ reported $\lambda$ of 1.002 ,

199 indicating a perfect control of inflation (from $\lambda=1.323$ in the ordinary permutation).

200 Visual inspection of the QQ plot also confirms the full control of stratification for

201 common variants (S2 Fig).

202 Estimate the number of clusters 
203 A naïve approach to randomize population-stratified samples is to group them into

204 ethnicity clusters and permute uniformly within each cluster [21-23]. The number of

205 clusters can be estimated similarly as estimating $\gamma$ for Bootstrat: we chose the smallest

206 number of clusters that sufficiently controls the stratification. Using the same desired

207 genomic control level ( $\lambda$ ranges between 1.010 and 0.990 ), we found that dividing the

208 samples into 8 clusters achieves the optimal genomic control (Table 1).

\section{Single common variant test}

210 Common variants were defined as variants with minor allele frequency (MAF) $>5 \%$.

21122,415 common variants are available in this study after QC. Parametric test adjusting

212 for PCs of ancestry is the de facto method for controlling the population stratification,

213 and has been demonstrated to work well $[8,10]$. We show that Bootstrat is able to

214 control the population stratification as good as the parametric test with PC adjustment

215 (Fig 2). Genomic inflation factors are 1.000 for parametric test with PCs, 1.002 for

216 Bootstrat with $\gamma=4,1.007$ for permutation within cluster ( 8 clusters), 0.912 for

217 BiasedUrn and 1.066 for EMMAX. Note that the purpose of this section is not to find the

218 best method in controlling stratification in single common variant tests. We consider

219 this a solved problem: parametric tests using PCs or $\operatorname{LMM}[18,19]$ have been proven to

220 be the solutions. We instead used this established solution as gold standard to estimate

221 parameters in other approaches, including Bootstrat and the permutation within cluster

222 approach. It is therefore not fair to use the single common variant test as the metric to

223 perform method comparison.

\section{Single rare variant test}


225 Rare variants were defined as variants with $\mathrm{MAF}<1 \%$ but with at least 10 minor alleles

226 (MAF $=0.25 \%$ in the current sample). There were 9,881 rare variants available in this

227 study after QC. Under the case/control study design, the test statistic distribution

228 among rare variants often fails to meet asymptotic assumptions of chi-square

229 distributed tests [26]. As a result, the corresponding $p$-value distribution lacks

230 uniformity, making visual inspection of the QQ plot uninformative and introducing

231 fundamental bias to measures of $\lambda$. To address this problem, we generated an empirical

232 null distribution of test statistics via permutation (see Methods:: Empirical null

233 distribution for single rare variant tests). These permutations are drawn independent

234 from population structure and have the advantage of conditioning on the sample size,

235 selected allele frequency distribution and case-control balance within the dataset. Both

236 the QQ plot and $\lambda$ can be recalibrated using this permuted null distribution.

237 Overall, every method reduces the genomic inflation present among rare

238 variants confounded by population stratification, whereby no control for population

239 stratification leads to a comparable level of genomic inflation as for common variants

$240(\lambda=1.356)$. Fig 3 shows the empirical QQ plot and corresponding median $\lambda$ statistic for

241 single rare variants under the variety of methods analyzed. Visual inspection of the QQ

242 plot against the asymptotic chi-square shows how the BiasedUrn test is generally

243 deflated across the distribution of test statistics, while the parametric test controlling

244 for the 1st PC shows marked deflation at the tail end of the distribution, suggesting a

245 lack of power due to the inclusion of a covariate into the regression. After recalibrating

246 observed test statistics to their permuted null distribution, tests generally track more 
247 closely to expectation across the board. While no test fully accounts for population

248 stratification among rare variants, Bootstrat outperforms all other tests when measuring

249 genomic inflation, matching the parametric test controlling for the 1st PC under the

250 asymptotic test distribution $(\lambda=1.047)$, and showing the lowest inflation using the

251 empirical test distribution $(\lambda=1.037)$.

\section{Gene-based rare variant test}

253 Variant-set Kernel Association Test (SKAT) $[2,12,16]$ uses a kernel machine to test for the

254 association between a set of SNPs within a gene and an outcome. The set of variants

255 that are tested together is defined based on variant characteristics such as frequency

256 and in silico functional class. SKAT using permutations for assessing significance was

257 modified to incorporate the ancestry informative randomization from Bootstrat. There

258 were 5,725 genes tested with an outcome simulated to have population structure using

2595 models: (1). SKAT with empirical significance, (2). SKAT with random permutation for

260 significance, (3). SKAT adjusted for PCs of ancestry as covariates, (4). SKAT adjusted for

261 PCs of ancestry as covariates with random permutation, and (5). SKAT modified with

262 Bootstrat. Inflation in the Q-Q plot was observed when no adjustment for population

263 has been performed (models 1 and 2), and adjusting for population structure by using

264 PCs of ancestry or Bootstrat more appropriately controls for the population structure

265 (Fig 4).

266 We assessed the type I error and power for the gene-based test using the 5

267 models described above. We found that type I error rates were severely inflated when

268 we did not account for the population structure (models 1 and 2). The inflation was not 
269 fully reduced when PCs of ancestry were used for adjustment of population structure

270 (Fig 5). In contrast, Bootstrat was able to better control the type I error at the

271 significance levels of 0.05, 0.01 and 0.005. For significance levels of 0.001 and 0.0005 ,

272 Bootstrat has similar performance as using PCs. Furthermore, power was found to be

273 similar across all the models at various nominal type I errors (Table 2). Note that

274 although Bootstrat has an additional parameter, this parameter was trained using single

275 common variant test and stayed the same across rare variant tests, including the single

276 variant test and the gene-based test. Therefore, we argue that the superior

277 performance of Bootstrat in testing rare variants is not due to overfitting.

278 Computational performance

279 Bootstrat was efficiently implemented: for our study cohort of 2,000 samples, it takes

2800.1 minutes to calculate the exchange probability matrix, and 8.8 minutes to perform

281 the permutations using this matrix (Table 3). This time is longer than the ordinary

282 permutation because the probabilistic sampling is more time consuming than sampling

283 with uniform probabilities. However, the time for running randomizations can be

284 reduced significantly (from 8.8 to 3.8 minutes in this study) for case/control study

285 designs using a more efficient design (Methods:: Efficient implementation for the case-

286 control study design). The exchange probability matrix only needs to be calculated

287 once per dataset, and can be reused with different phenotypes and parameters. In

288 practice, the run time can be further reduced by using adaptive permutations $[16,24]$

289 and parallelization platforms such as MapReduce $[25,29]$. 


\section{Discussion}

292 We have presented Bootstrat, a bootstrap based randomization scheme that corrects

293 for population stratification in testing rare variants for association with diseases. We

294 compared this scheme with existing methods including EMMAX, BiasedUrn,

295 permutation within cluster and SKAT and found that Bootstrat controls the type I error

296 better than existing methods, while keeping the power at similar levels.

297 In our comparison, we did not include the recently developed KM method, which

298 is an extension of SKAT to accommodate related subjects from multiple independent

299 families $[26,30]$. The authors of the KM test only evaluated their method for common

300 variants in SNP sets with families, while we propose to use Bootstrat when there are

301 multiple sub-population groups within the data. Therefore, comparison with KM

302 method is beyond the scope of this paper.

303 Bootstrat is compatible with both case/control studies and quantitative traits

304 and works with most existing rare variant tests. Users have the option to use this

305 permutation scheme to run any available tests implemented in PLINK. We have

306 implemented this permutation scheme in PLINK, and R code that implements this

307 scheme in the SKAT framework is available upon request.

309 Methods

310 Dataset

311 Both cohorts used in this study were population controls genotyped on the exome chip.

312 After QC and removing related samples, the NIMH control cohort has 1,036 samples and 
313230,329 variants [31]; and the Swedish cohort has 6,063 samples and 246,843 variants

314 [32]. After merging, we kept 199,928 autosomal variants available in both cohorts. We

315 randomly selected 1,000 samples from each cohort to create the dataset used in this

316 analysis (Fig 1).

317 Remove related samples

318 We performed LD prune on the common variants (minor allele frequency $>5 \%$ ) using

319 PLINK $[27,33]$. These LD pruned common variants were then used to calculate the

320 Identity-by-descent (IBD) matrix. Using the IBD matrix, we identified 139 related sample

321 pairs $(\mathrm{Pi}$ hat $>0.2)$ in the Swedish cohort. No related samples were found within the

322 NIMH cohort or across the two cohorts. In the Swedish cohort, we randomly removed

323 one sample in each pair, a total of 134 unique samples, to create a dataset of

324 independent samples.

\section{Bootstrat}

326 Individuals having the same genetic ethnicity have been shown to cluster closer in the

327 principal components (PCs) space. Many genome-wide association studies (GWAS) used

328 PCs as covariates to correct for population stratification. Various measures have been

329 used to choose which PCs to include in the analyses such as the visual inspection of the

330 QQ plot, the genomic inflation factor, and testing the associations between PCs and

331 case/control. In the following context, we assume users know the appropriate PCs to

332 use from the ordinary GWAS. Denote the $\mathrm{p}$-th PC as $w_{p}$ and its eigenvalue as $\lambda_{p}$, we

333 calculated the pairwise Euclidean distance between samples $i$ and $j$ as 


$$
d_{i j}=\sqrt{\sum_{p} \lambda_{p}\left(w_{p i}-w_{p j}\right)^{2}}
$$

336 calculated the raw pairwise exchange probability from this distance using the

337 exponential transformation $E_{i j}=\exp \left(-2^{\gamma} d_{i j}\right)$ and normalized the raw probability to

338 obtain the final probability for the weighted bootstrap. Other transformations,

339 including linear and inverse, were also considered but we found the exponential

340 transformation provided the most robust results (results not shown). For sample $i$, the

341 probability to draw the phenotype from sample $j$ is

$$
\begin{aligned}
\operatorname{Pr}(j \mid i) & =E(i, j) / \sum_{j^{\prime}} E\left(i, j^{\prime}\right) \\
& =\frac{\exp \left(-2^{\gamma} d_{i j}\right)}{\sum_{j^{\prime} \in\{\text { all }\}} \exp \left(-2^{\gamma} d_{i j^{\prime}}\right)}
\end{aligned}
$$

344 samples in the studies, including sample $i$ itself. $\gamma$ is a scaling parameter that controls

345 how the probability scales with the distance, which can take any value between - $\infty$ and

$346+\infty . y=-\infty$ gives uniform exchange probability for each sample pair and is essentially

347 the ordinary bootstrap. $y=+\infty$ confines each sample to only swapping with itself. We

348 performed a grid search to find the optimal $\gamma$ based on the genomic inflation factor

349 using common variants. This is computationally feasible because only the LD pruned

350 common variants (less than 20,000 variants in exome chip) are needed to calculate the

351 inflation factor.

352 We calculated the test statistics in each bootstrap and created a null distribution

353 for each variant. The empirical $p$-value of an association is defined as the fraction of 
354 time a better or equal test statistic was achieved under the null. In practice, we

355 performed bootstrap in an adaptive manner to save computation time [28,33].

\section{Efficient implementation for the case-control study design}

357 For case-control study design, instead of performing bootstrap for each sample, a more

358 efficient method would be to calculate the probability for a particular sample to be case

359 or control, i.e.,

$$
\operatorname{Pr}(\text { case } \mid i)=\frac{\sum_{j^{\prime} \in\{\text { case }\}} \exp \left(-2^{\gamma} d_{i j^{\prime}}\right)}{\sum_{j^{\prime} \in\{\text { all }\}} \exp \left(-2^{\gamma} d_{i j^{\prime}}\right)},
$$

361 and use this probability to simulate the case/control status from a binomial distribution.

\section{Baseline methods}

363 As the comparison baseline, we performed logistic regression with and without the PCs

364 as covariates, and permutation tests with a minimum of 1,000 permutations in PLINK

$3651.08[1,3,33]$. We performed a linear mixed model implementation, EMMAX, using the

366 package released on March 2007 with default parameters [4,19], and Biased Urn using

367 the R 'BiasedUrn' package in CRAN with recommended revisions from the paper

$368[8,10,24]$. Other methods discussed are:

369 Permutation within cluster. This approach divides samples into clusters based on their

370 genetic similarity. In this study, the similarity was calculated as the Euclidian distance

371 using the 1st principal component. Within each cluster, we assume population

372 homogeneity and shuffle samples with uniform probability. Clusters with only case or

373 control samples are ignored. The number of clusters was chosen such that the genomic

374 control has achieved a satisfactory level (Results::Estimate the number of clusters). 
375 SKAT. We used The Sequence Kernel Association Test (SKAT) [12] version 0.81

376 (implemented in R) to for the gene-based tests using an identity-by-state kernel. SKAT

377 evaluates qualitative and quantitative traits by comparing rare variant similarity with

378 phenotypic similarity, thus permitting variants to be associated with an increase and

379 decrease in the phenotype within a gene.

\section{Empirical null distribution for single rare variant tests}

381 To generate an empirical null distribution for single rare variant tests, phenotype

382 permutation was performed using PLINK, where case/control labels were randomly re-

383 assigned in each permutation. By using the PLINK command --mperm-save-all, we saved

384 all permuted test statistics. These statistics were combined together into a single vector,

385 ordered, and split into bins where the size of each bin was the number of permutations.

386 To average across permutations, the median $p$-value was pulled from each bin. We used

387100 permutations to derive the empirical null.

\section{Type I Error and Power for gene-based tests}

389 We assessed type I error and power for gene-based tests using the following 5 models:

390 1) SKAT with empirical significance, 2) SKAT with random permutation for significance,

391 3) SKAT adjusting for PCS of ancestry as covariates, 4) SKAT adjusting for PCs of ancestry

392 as covariates with random permutation for significance, and 5) SKAT modified with

393 Bootstrat. To simulate the effect of population structure, we randomly selected $70 \%$ of

394 American samples and $30 \%$ of Swedish samples as cases and set the remaining samples

395 to be controls. Type I error was assessed at the 0.05 with 1,000 iterations and at lower

396 significance levels $(0.01,0.005,0.001$ and 0.0005$)$ with 20,000 iterations under the null 
397 models (odds ratio of 1). Power was assessed at the 0.05 and 0.01 significance levels

398 with 1,000 iterations for each model. We assigned each variant to be neutral or have an

399 effect with a $30 \%$ (or $50 \%$ ) probability and simulated the phenotype based on the

400 genotypes using odds ratios of 2.5 and 5 . We randomly sampled genes on chromosome

4011 for both type I error and power assessments.

402

\section{Acknowledgments}

404 GMP is supported by the National Heart, Lung, And Blood Institute of the National

405 Institutes of Health under Award Number K01HL125751. The content is solely the

406 responsibility of the authors and does not necessarily represent the official views of the

407 National Institutes of Health. BMN acknowledges funding from the Gerstner family

408 foundation. BR and KB acknowledge funding from the Max Planck Society. Carl-Johann

409 SIMON-GABRIEL is supported by a Google European Doctoral Fellowship in Causal

410 Inference. The funders had no role in study design, data collection and analysis, decision

411 to publish, or preparation of the manuscript. 


\section{List of figures}

413 Fig 1. Population stratification was simulated using Swedish and American control

414 cohort.

415 (a) Case and control statuses were simulated for Swedish and American cohorts

416 (Methods). The first two principal components were plotted, although PC1 alone was

417 sufficient to control the population stratification (Results::Principal components). We

418 traced 100 randomizations of two randomly picked samples (as back crosses and

419 asterisks respectively) as an illustration, showing that samples are shuffled only with

420 other samples that have close genetic distance. (b) The exchange probability for the

421 two randomly picked samples decreases with the genetic distance.

422 Fig 2. QQ plot of common variants.

423 QQ plot of the test statistics of common variants for various methods discussed. For

424 methods with parameters, we used the parameter that achieves the best performance

425 (Results): we used $\gamma=4$ for Bootstrat and 8 clusters for permutation within cluster.

426 Fig 3. QQ plot of rare variants.

427 QQ plot for various methods calibrated using the null phenotype. Only rare variants

428 were plotted. We used the same parameters as for common variants: $\gamma=4$ for Bootstrat

429 and 8 clusters for permutation within cluster.

430 Fig 4. QQ plot of gene-based tests under the null.

431 QQ plot of the test statistics of gene-based tests. "SKAT, no Adj": SKAT with empirical

432 significance. "SKAT, no Adj (Perm)": SKAT with random permutation for significance.

433 "SKAT, PC Adj": SKAT adjusting for PC1 as a covariate. "SKAT, PC Adj (Perm)": SKAT 
bioRxiv preprint doi: https://doi.org/10.1101/068999; this version posted August 11,2016 . The copyright holder for this preprint (which was not

certified by peer review) is the author/funder, who has granted bioRxiv a license to display the preprint in perpetuity. It is made available under aCC-BY-NC-ND 4.0 International license.

434 adjusting for PC1 as a covariate with random permutation for significance. "Bootstrat":

435 SKAT modified with Bootstrat.

436 Fig 5. Type I error of gene-based tests using rare variants.

437 Type I error was calculated as the proportion of genes meeting the specified $\alpha$ level

438 (color coded) across all the genes analyzed. Dashed lines indicate the expected type I

439 error and open circles indicate the observed type I error for each method. 


\section{$440 \quad$ List of Tables}

441 Table 1. Genomic inflation factor using common variants.

\begin{tabular}{|c|c|c|c|c|c|}
\hline Method & Parameter & Lambda & Method & Parameter & Lambda \\
\hline \multirow[t]{4}{*}{ Parametric } & No PC used & 1.330 & BiasedUrn & - & 0.912 \\
\hline & PC1 & 1.000 & EMMAX & - & 1.066 \\
\hline & PC1-2 & 1.010 & \multirow[t]{12}{*}{ Bootstrat } & $\gamma=-3$ & 1.323 \\
\hline & PC1-3 & 0.992 & & $\gamma=-2$ & 1.323 \\
\hline \multirow{10}{*}{$\begin{array}{l}\text { Permutation } \\
\text { within Cluster }\end{array}$} & $k=1$ & 1.323 & & $\gamma=-1$ & 1.289 \\
\hline & $k=2$ & 1.031 & & $\nu=0$ & 1.222 \\
\hline & $k=3$ & 1.035 & & $v=1$ & 1.098 \\
\hline & $k=4$ & 1.021 & & $v=2$ & 1.026 \\
\hline & $k=5$ & 1.030 & & $\gamma=3$ & 1.012 \\
\hline & $k=6$ & 1.035 & & $v=4$ & 1.002 \\
\hline & $k=7$ & 1.025 & & $\gamma=5$ & 1.007 \\
\hline & $k=8$ & 1.007 & & $v=6$ & 1.007 \\
\hline & $k=9$ & 1.016 & & $v=7$ & 1.002 \\
\hline & $k=10$ & 1.016 & & $y=8$ & 0.993 \\
\hline
\end{tabular}

$442 \mathrm{k}$ is the number of clusters used in the permutation within cluster approach. $v$ is the 443 parameter used in Bootstrat (Methods::Bootstrat). 
444 Table 2. Power for gene-based tests using rare variants

\begin{tabular}{|r|r|l|l|l|c|c|c|}
\hline OR & $\begin{array}{l}\text { Prop. } \\
\text { func. }\end{array}$ & $\alpha$ & No adj & $\begin{array}{c}\text { No adj } \\
\text { (perm) }\end{array}$ & PC adj & $\begin{array}{c}\text { PC adj } \\
\text { (perm) }\end{array}$ & Boostrat \\
\hline 2.5 & 0.3 & 0.05 & $0.51 \pm 0.03$ & $0.52 \pm 0.03$ & $0.45 \pm 0.03$ & $0.45 \pm 0.03$ & $0.42 \pm 0.03$ \\
\hline 2.5 & 0.3 & 0.01 & $0.36 \pm 0.03$ & $0.37 \pm 0.03$ & $0.34 \pm 0.03$ & $0.34 \pm 0.03$ & $0.30 \pm 0.03$ \\
\hline 2.5 & 0.5 & 0.05 & $0.66 \pm 0.03$ & $0.66 \pm 0.03$ & $0.65 \pm 0.03$ & $0.65 \pm 0.03$ & $0.58 \pm 0.03$ \\
\hline 2.5 & 0.5 & 0.01 & $0.54 \pm 0.03$ & $0.53 \pm 0.03$ & $0.52 \pm 0.03$ & $0.52 \pm 0.03$ & $0.45 \pm 0.03$ \\
\hline 5 & 0.3 & 0.05 & $0.70 \pm 0.03$ & $0.70 \pm 0.03$ & $0.68 \pm 0.03$ & $0.68 \pm 0.03$ & $0.63 \pm 0.03$ \\
\hline 5 & 0.3 & 0.01 & $0.60 \pm 0.03$ & $0.60 \pm 0.03$ & $0.59 \pm 0.03$ & $0.59 \pm 0.03$ & $0.53 \pm 0.03$ \\
\hline 5 & 0.5 & 0.05 & $0.86 \pm 0.02$ & $0.85 \pm 0.02$ & $0.86 \pm 0.02$ & $0.86 \pm 0.02$ & $0.81 \pm 0.02$ \\
\hline 5 & 0.5 & 0.01 & $0.77 \pm 0.03$ & $0.78 \pm 0.03$ & $0.77 \pm 0.03$ & $0.77 \pm 0.03$ & $0.73 \pm 0.03$ \\
\hline
\end{tabular}

445 Power was assessed using an outcome simulated with population structure and an odds

446 ratio of 2.5 and 5 (Methods::Type I Error and Power for gene-based tests). Prop.

447 functional is the proportion of variants that are functionally related to the outcome. We 448 assigned each variant to be neutral or have an effect with a $30 \%$ (or $50 \%$ ) probability. A 449 greater proportion of functional variants simulates less noise in the gene-based 450 association testing. $\alpha$ is the type I error at which the power was assessed. Power and its 451 standard error were both reported. 
453 Table 3. Run time for each method

\begin{tabular}{|r|r|r|r|}
\hline Method & Preparation time & Analysis time & Total \\
\hline Parametric & - & 0.4 & 0.4 \\
\hline BiasedUrn & - & 335 & 335 \\
\hline EMMAX & 5.8 & 1.0 & 6.8 \\
\hline Permutation within cluster & 0.0 & 3.3 & 3.3 \\
\hline Bootstrat & $\mathbf{0 . 1}$ & $\mathbf{8 . 8}$ & $\mathbf{8 . 9}$ \\
\hline Bootstrat (case/control) & 0.1 & 3.8 & 3.9 \\
\hline SKAT with PC adjustment & & & \\
and permutations & - & 23.0 & 23.0 \\
\hline SKAT with Bootstrat & 0.1 & 33.2 & 33.3 \\
\hline
\end{tabular}

454 CPU time (in minutes) for performing tests on chromosome 1. 'Preparation time' is the

455 time for pre-calculating inputs for some methods, including the kinship matrix for

456 EMMAX, clustering for 'permutation within cluster', and exchange probability matrix for

457 Bootstrat. This step only needs to be done once per cohort and can be reused in analysis

458 with different phenotypes and options. 'Analysis time' is the time for actually

459 performing the association study given the pre-calculations. 


\section{References}

461 1. Good P. Exchangeable random variables. 2010;26: 2214-author reply

462 2215. doi:10.1093/bioinformatics/btq297

463 2. Jostins L, Ripke S, Weersma RK, Duerr RH, Mcgovern DP, Hui KY, et al.

$464 \quad$ Host-microbe interactions have shaped the genetic architecture of

465 inflammatory bowel disease. Nature. Nature Publishing Group; 2012;491: 119-124. doi:10.1038/nature11582

3. Good PI. Permutation, Parametric, and Bootstrap Tests of Hypotheses. Springer Science \& Business Media; 2006.

4. Pirinen M, Donnelly P, Spencer CCA. Including known covariates can reduce power to detect genetic effects in case-control studies. Nat Genet. 2012;44: 848-. doi:10.1038/ng.2346

5. Do R, Stitziel NO, Won H-H, Jørgensen AB, Duga S, Angelica Merlini P, et al. Exome sequencing identifies rare LDLR and APOA5 alleles conferring risk for myocardial infarction. Nature. Nature Publishing Group; 2014;:- . doi:10.1038/nature13917

6. CARDIoGRAMplusC4D Consortium, Deloukas P, Kanoni S, Willenborg C, Farrall M, Assimes TL, et al. Large-scale association analysis identifies new risk loci for coronary artery disease. Nat Genet. 2013;45: 25-33. doi:10.1038/ng.2480

7. Arking DE, Pulit SL, Crotti L, van der Harst P, Munroe PB, Koopmann TT, et al. Genetic association study of QT interval highlights role for calcium signaling pathways in myocardial repolarization. Nat Genet. Nature Publishing Group; 2014;:-. doi:10.1038/ng.3014

8. Price AL, Patterson NJ, Plenge RM, Weinblatt ME, Shadick NA, Reich D. association studies. Nat Genet. 2006;38: 904-909. doi:10.1038/ng1847

9. Ripke S, O'Dushlaine C, Chambert K, Moran JL, Kähler AK, Akterin S, et al. Genome-wide association analysis identifies 13 new risk loci for schizophrenia. Nat Genet. Nature Publishing Group; 2013;45: 1150-1159. doi:doi:10.1038/ng.2742

10. Price AL, Zaitlen NA, Reich D, Patterson N. New approaches to population stratification in genome-wide association studies. Nat Rev Genet. 2010;11: 459-463. doi:10.1038/nrg2813

11. Ripke S, Neale BM, Corvin A, Walters JTR, Farh K-H, Holmans PA, et al. Biological insights from 108 schizophrenia-associated genetic loci. Nature. 2014;511: 421-427. doi:10.1038/nature13595 
497

498

499

500

501

502

503

504

505

506

507

508

509

510

511

512

513

514

515

516

517

518

519

520

521

522

523

524

525

526

527

528

529

530

531

532

533
12. Wu MC, Lee S, Cai T, Li Y, Boehnke M, Lin X. Rare-variant association testing for sequencing data with the sequence kernel association test. Am J Hum Genet. 2011;89: 82-93. doi:10.1016/j.ajhg.2011.05.029

13. Li B, Leal SM. Methods for detecting associations with rare variants for common diseases: application to analysis of sequence data. Am J Hum Genet. 2008;83: 311-321. doi:10.1016/j.ajhg.2008.06.024

14. Neale BM, Rivas MA, Voight BF, Altshuler D, Devlin B, Orho-Melander M, et al. Testing for an Unusual Distribution of Rare Variants. PLoS Genet. Public Library of Science; 2011;7: e1001322. doi:10.1371/journal.pgen.1001322

15. Listgarten J, Lippert C, Kang EY, Xiang J, Kadie CM, Heckerman D. A powerful and efficient set test for genetic markers that handles confounders. Bioinformatics. 2013;29: 1526-1533.

doi:10.1093/bioinformatics/btt177

16. Ronglin Che JRJAAM-RCCB. An adaptive permutation approach for genome-wide association study: evaluation and recommendations for use. BioData Mining. BioMed Central; 2014;7: 9. doi:10.1186/1756-0381-7-9

17. McVean G, Mathieson I. Differential confounding of rare and common variants in spatially structured populations. Nat Genet. Nature Publishing Group; 2012;: 1-6. doi:10.1038/ng.1074

18. Kang HM, Zaitlen NA, Wade CM, Kirby A, Heckerman D, Daly MJ, et al. Efficient control of population structure in model organism association mapping. Genetics. 2008;178: 1709-1723.

doi:10.1534/genetics.107.080101

19. Kang HM, Sul JH, Service SK, Zaitlen NA, Kong S-Y, Freimer NB, et al. Variance component model to account for sample structure in genomewide association studies. Nat Genet. 2010;42: 348-354. doi:10.1038/ng.548

20. Devlin B, Roeder K. Genomic control for association studies. Biometrics. Wiley Online Library; 1999;55: 997-1004.

21. Pritchard JK, Stephens M, Donnelly P. Inference of population structure using multilocus genotype data. Genetics. 2000;155: 945-959.

22. Pritchard JK, Stephens M, Rosenberg NA, Donnelly P. Association mapping in structured populations. AJHG. 2000;67: 170-181. doi:10.1086/302959

23. Pritchard JK, Donnelly P. Case-Control Studies of Association in Structured or Admixed Populations. Theoretical population biology. 2001. 
534 24. Epstein MP, Duncan R, Jiang Y, Conneely KN, Allen AS, Satten GA. A

535

536

537

538

539

540

541

542

543

544

545

546

547

548

549

550

551

552

553

554

555

556

557

558

559

560

561

562

563

564

565

566

567

568

permutation procedure to correct for confounders in case-control studies, including tests of rare variation. Am J Hum Genet. 2012;91: 215-223. doi:10.1016/j.ajhg.2012.06.004

25. Fog A. Sampling Methods for Wallenius" and Fisher"s Noncentral Hypergeometric Distributions. Communications in Statistics - Simulation and Computation. 2008;37: 241-257. doi:10.1080/03610910701790236

26. Bigdeli TB, Neale BM, Neale MC. Statistical Properties of Single-Marker Tests for Rare Variants. Twin Research and Human Genetics. Cambridge University Press; 2014;17: 143-150. doi:10.1017/thg.2014.17

27. Good PI. Extensions of the concept of exchangeability and their applications. Journal of Modern Applied Statistical Methods. 2002.

28. Barbe P, Bertail P. The Weighted Bootstrap. Springer Science \& Business Media; 1995.

29. Huang H, Tata S, Prill RJ. BlueSNP: R package for highly scalable genome-wide association studies using Hadoop clusters. 2013;29: 135136. doi:10.1093/bioinformatics/bts647

30. Schifano ED, Epstein MP, Bielak LF, Jhun MA, Kardia SLR, Peyser PA, et al. SNP Set Association Analysis for Familial Data. Genet Epidemiol. 2012;: n/a-n/a. doi:10.1002/gepi.21676

31. Shi J, Levinson DF, Duan J, Sanders AR, Zheng Y, Pe'er I, et al. Common variants on chromosome 6p22.1 are associated with schizophrenia. Nature. 2009;460: 753-757. doi:10.1038/nature08192

32. Szatkiewicz JP, O'Dushlaine C, Chen G, Chambert K, Moran JL, Neale $\mathrm{BM}$, et al. Copy number variation in schizophrenia in Sweden. Molecular Psychiatry. 2014;19: 762-773. doi:10.1038/mp.2014.40

33. Purcell S, Neale B, Todd-Brown K, Thomas L, Ferreira MAR, Bender D, et al. PLINK: A Tool Set for Whole-Genome Association and PopulationBased Linkage Analyses. The American Journal of Human Genetics. 2007;81: 559-575. doi:10.1086/519795

\section{Supporting Information}

S1 Fig. Selection of the principal components. QQ plot of the test statistics of common variants when various numbers of principal components were used.

S2 Fig. Selection of the Bootstrat parameter. QQ plot of the test statistics of common variants for $\gamma$ ranges between -3 and 8 . 
bioRxiv preprint doi: https://doi.org/10.1101/068999; this version posted August 11,2016 . The copyright holder for this preprint (which was not certified by peer review) is the author/funder, who has granted bioRxiv a license to display the preprint in perpetuity. It is made available under aCC-BY-NC-ND 4.0 International license.

\section{S1 File. Psuedocode for estimating the Bootstrat parameter.}


a

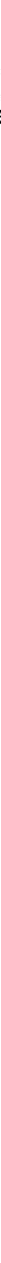

b

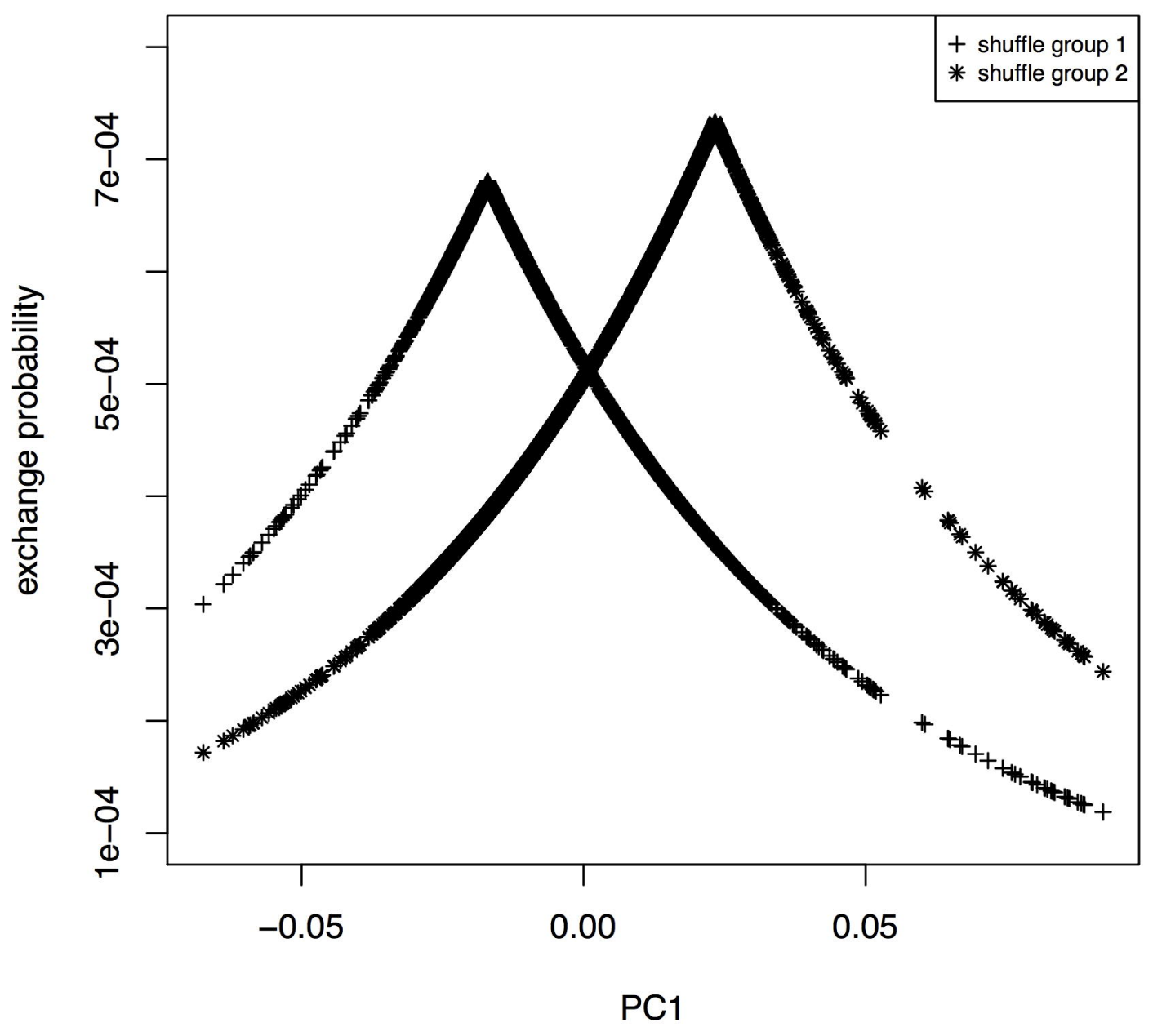


Parametric with 1 PC
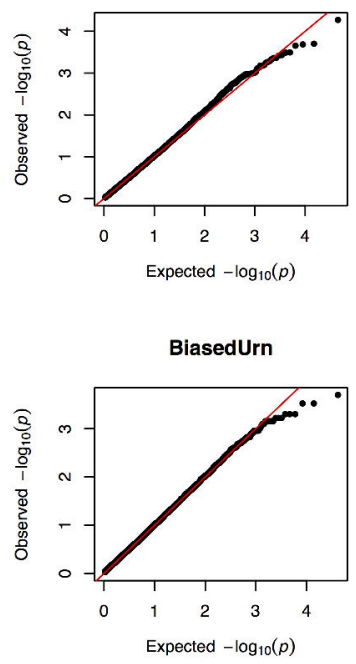

\section{Bootstrat}

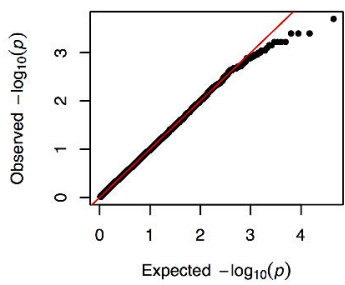

Emmax

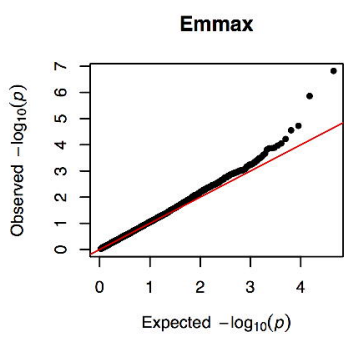

\section{Permutation within cluster}

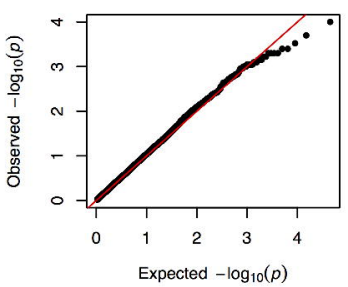




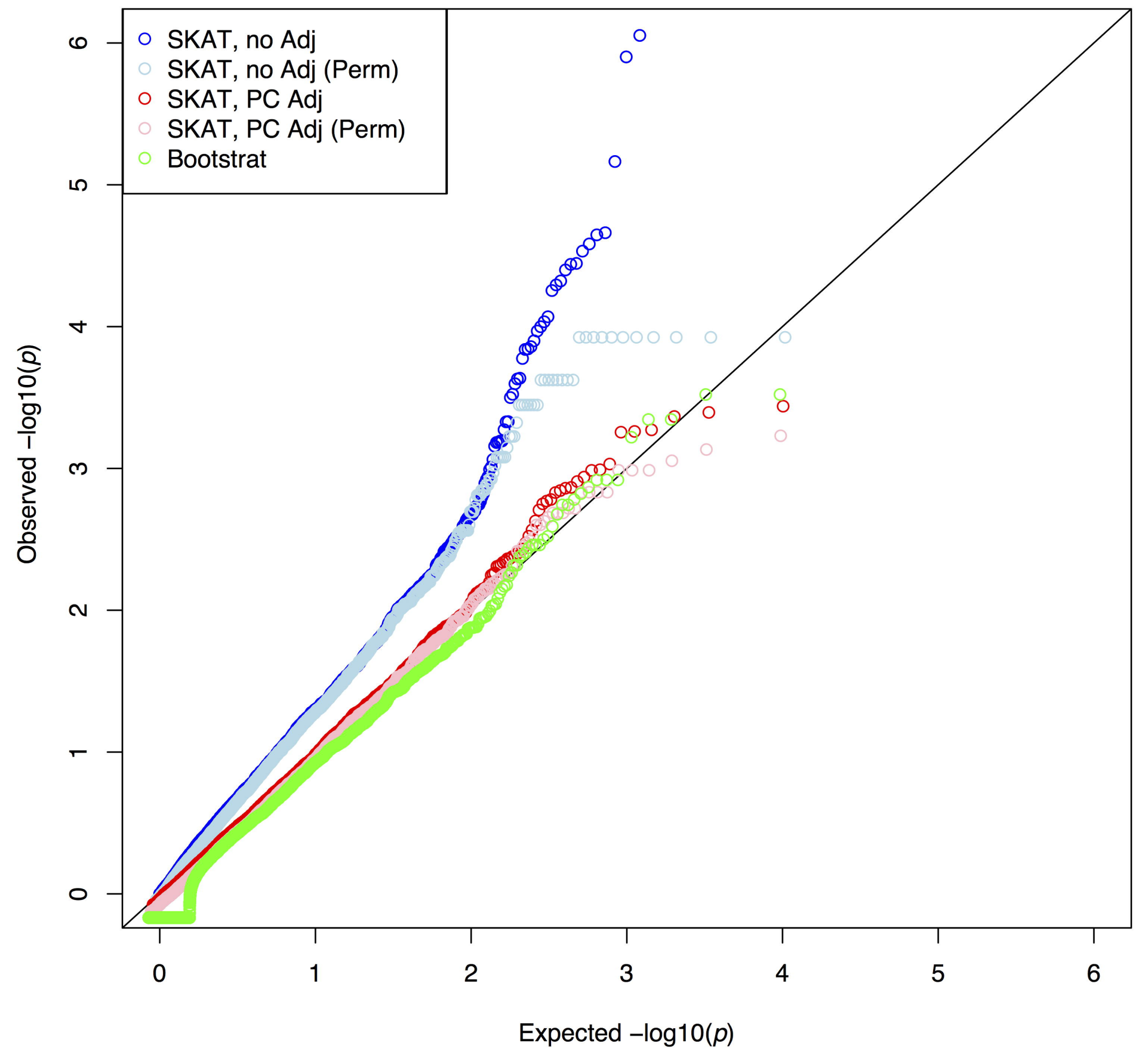


